\title{
Diversifying the biomedical workforce during the COVID-19 pandemic
}

To the Editor - While the fallout from COVID-19 has wrought widespread hardships, early-career underrepresented minority (URM) people within the biomedical training pipeline are particularly vulnerable to the disruptions caused by this pandemic. Lost opportunities, such as the cancellation of over half of all 2020 summer internships ${ }^{1}$, can have long-lasting consequences on career trajectories. This may widen gaps in representation that have contributed to the disproportionate toll of COVID-19 on people of color ${ }^{2-4}$. URM trainees already experience barriers to meaningful work in research, such as limited early exposure to health careers and the preponderance of unpaid or low-wage internships ${ }^{5}$. They may also have fewer resources to withstand the negative impacts of the pandemic. In the current global discourse on social justice and systemic inequities, leading institutions must promote an inclusive workforce.

To meet this need, we created the Massachusetts General Hospital COVID Corps Research Internship (https://www.monganinstitute.org/ covid-corps-2020-cohort), pairing undergraduate and medical students with investigators working on COVID-19 research. To keep trainees safe, mentors supervised projects remotely. We offered a stipend through in-kind support and prioritized the selection of mentorship teams with experienced senior researchers and accessible junior faculty able to provide day-to-day oversight. Where possible, mentors funded their interns, which allowed us to triple our intended cohort size.

Two weeks after the inception of our program, hospital administration lowered barriers related to hiring, shortening the time from offer to start date to fewer than three weeks. Program leadership provided administrative oversight and managed research ethics and training on electronic health records, while the remote experience allowed us to safely bypass an institution-wide hiring freeze, which allowed investigators to quickly launch COVID-19 projects. We hosted regular office hours to build a sense of community during a time of social distancing and conducted networking events and seminars on topics such as applying to graduate school and making a good first impression. Interest in the program exceeded our most optimistic projections, with 238 applications in six days, a response so swift that we closed the application process early.

To identify a diverse pool of candidates, we collaborated with the Massachusetts General Hospital Disparities Solutions Center to access their extensive network of partnering institutions and targeted pre-health students actively seeking workstudy opportunities. With this emphasis on diversity, we welcomed an inaugural class of 22 people, $100 \%$ of whom are from groups underrepresented in science: 16 identify as female, non-binary, questioning or transgender; 18 are first-generation university students; 13 are immigrants or new Americans; and 18 shared details of an economically disadvantaged background. Eight were Black or African-American, four were LatinX and nine were Asian. Their mean undergraduate GPA was 3.86. In confidential exit interviews, 16 interns conveyed their intention to continue working with their mentors during the academic year, some of whom will be seeking funding through the US National Institutes of Health's Research Supplements to Promote Diversity in Health-Related Research; $91 \%$ of the interns agreed that being part of a diverse cohort enhanced their program experience.

Assertions of a limited pipeline of interested URMs did not prove valid. Instead, we highlight the need for professional opportunities for qualified and talented URMs and demonstrate that it is possible to adapt mentorship programs to fit this new environment. Institutions should work deliberately toward a biomedical workforce that better represents the country it serves. In the midst of a pandemic and a long-overdue activist movement on racial inequality, we deployed an engaged workforce ready to address both problems. We call upon others to do the same.

Long H. Nguyen (D) 1,2,

Aswita Tan-McGrory ${ }^{3,4}$, Alice Y. Oh ${ }^{3}$, Esteban A. Barreto ${ }^{3}$, Stephen J. Bartels ${ }^{3}$, Katrina A. Armstrong ${ }^{5}$, Andrew T. Chan ${ }^{1,2}$ and Erica T. Warner (D) 2,3,5

${ }^{1}$ Division of Gastroenterology, Massachusetts General Hospital and Harvard Medical School, Boston, MA, USA. ${ }^{2}$ Clinical and Translational Epidemiology Unit, Massachusetts General Hospital and Harvard Medical School, Boston, MA, USA. ${ }^{3}$ Mongan Institute, Massachusetts General Hospital and Harvard Medical School, Boston, MA, USA. ${ }^{4}$ Disparities Solutions Center, Massachusetts General Hospital and Harvard Medical School, Boston, MA, USA. ${ }^{5}$ Department of Medicine, Massachusetts General Hospital and Harvard Medical School, Boston, MA, USA.

$\bigotimes_{e-m a i l: \text { ewarner@mgh.harvard.edu }}$

Published online: 30 October 2020 https://doi.org/10.1038/s41591-020-1134-7

References

1. Stansell, A. Glassdoor Economic Research https://www.glassdoor. com/research/internship-hiring-coronavirus/ (2020).

2. Price-Haywood, E. G., Burton, J., Fort, D. \& Seoane, L. N. Engl. J. Med. 382, 2534-2543 (2020).

3. Lo, C.-H. et al. medRxiv https://doi. org/10.1101/2020.06.18.20134742 (2020).

4. Millett, G. A. et al. Ann. Epidemiol. 47, 37-44 (2020).

5. Toretsky, C., Mutha, S. \& Coffman, J. Healthforce Center at UCSF https://healthforce.ucsf.edu/sites/healthforce.ucsf. edu/files/publication-pdf/Breaking\%20Barriers $\% 20$ for $\% 20$ Underrepresented $\% 20$ Minorities $\% 20$ in $\% 20$ the $\% 20$ Health $\% 20$ Professions\%20.pdf (2018).

Author contributions

Concept and design, L.H.N., A.T.C. and E.T.W.; data acquisition, L.H.N., A.T.-M., A.Y.O., E.A.B., A.T.C. and E.T.W.; drafting of the manuscript, L.H.N., A.T.C. and E.T.W.; critical revision of the manuscript, all authors.

Competing interests

The authors declare no competing interests. 